\title{
OPEN Negative impact of the hypopnea index or duration increase after a non-frame work surgery in patients with very severe obstructive sleep apnea
}

\author{
Ethan I. Huang ${ }^{1,2,3 凶}$, Shu-Yi Huang ${ }^{2,4,5}$, Yu-Ching Lin ${ }^{2,3,4,6}$, Chieh-Mo Lin ${ }^{4,7}$, Chin-Kuo Lin ${ }^{4,7}$, \\ Ying-Chih Huang ${ }^{8}$, Chia-Yu Hsu ${ }^{8}$ \& Jian-An Su ${ }^{9}$
}

A non-framework surgery could change the postoperative components of breathing disturbances and increase the frequency or duration of hypopnea in patients with very severe obstructive sleep apnea (OSA). Either an increase of hypopnea index, which increases apnea-hypopnea index (AHI), or an increase of its duration raises the concern of worsening the oxygen desaturation and so morbidity and mortality associated with OSA. It is unclear how the oxygen saturation would change in those having increased frequency or duration of hypopneas after the surgery. Here in 17 patients with $A H I \geq 60$ events/h, having increased frequency or duration of hypopneas after the non-framework surgery, the results show that the surgery improved oxygen saturation by reducing obstructive-apnea index ( 36.1 events/h) and duration ( $8.6 \mathrm{~s} / \mathrm{event})$, despite it increased hypopnea index $(16.8$ events $/ \mathrm{h})$ and duration $(9.8 \mathrm{~s} / \mathrm{event})$. The surgery improved the average of the mean oxyhemoglobin saturation of pulse oximetry (SpO2) by $2.8 \%$ (toward a ceiling mean of $94.3 \%$ ), mean minimal SpO2 by $7.5 \%$, and mean desaturation by $5 \%$. The results suggest sufficient apnea reduction and shift from apnea to hypopnea may mask the negative impact of the increase of hypopnea index or duration and improve postoperative mean $\mathrm{SpO}$, minimal $\mathrm{SpO}$, and mean desaturation.

Hypopnea is a part of AHI, which serves as a disease- and severity-defining metric for OSA ${ }^{1}$. In our earlier works, the hypopnea ratio in AHI may be increased after a non-framework surgery ${ }^{2}$ by decreasing apneas or converting apneas into hypopneas in patients with very severe $\mathrm{OSA}^{3,4}$. Here a non-framework surgery is defined as a procedure that does not involve the skeletal framework of the upper airway, i.e., maxilla and mandible. An increase of hypopnea ratio or duration raises the concern of worsening the oxygen desaturation associated with the disease.

Oxygen desaturation is associated with event duration ${ }^{5-8}$. For both components (of apnea and hypopnea), OSA patients with long average apnea-hypopnea duration had worse mean oxygen saturation percentage than the patients with short average duration ${ }^{5}$. For apneas, patients with a long mean apnea duration had worse blood oxygen levels and sleep parameters ${ }^{6}$. For hypopneas, increased hypopnea duration may worsen $\mathrm{SpO} 2$ desaturation, but hypopneas were reported to lead to less severe $\mathrm{SpO} 2$ desaturation compared to obstructive apneas ${ }^{7}$. A similar study by Yilmaz Durmaz and Gunes reported that longer mean hypopnea duration was related to lower mean oxygen saturation but was not associated with an oxygen desaturation event below $85 \%{ }^{8}$. The common ground is that the worse oxygen desaturation can come from more events or longer duration of hypopnea. The effect of hypopneas on oxygen desaturation is smaller than that of obstructive apneas.

${ }^{1}$ Department of Otolaryngology, Chang Gung Memorial Hospital, Chiayi 61363, Taiwan, ROC. ${ }^{2}$ Sleep Center, Chang Gung Memorial Hospital, Chiayi 61363, Taiwan, ROC. ${ }^{3}$ Department of Medicine, School of Medicine, Chang Gung University, Taoyuan 33302, Taiwan, ROC. "Division of Pulmonary and Critical Care Medicine, Chang Gung Memorial Hospital, Chiayi 61363, Taiwan, ROC. ${ }^{5}$ Department of Nursing, Chang Gung University of Science and Technology, Chiayi 61363, Taiwan, ROC. ${ }^{6}$ Department of Respiratory Care, Chang Gung University of Science and Technology, Chiayi 61363, Taiwan, ROC. ${ }^{7}$ Graduate Institute of Clinical Medical Sciences, College of Medicine, Chang Gung University, Taoyuan 33302, Taiwan, ROC. ${ }^{8}$ Department of Neurology, Chang Gung Memorial Hospital, Chiayi 61363, Taiwan, ROC. ' Department of Psychiatry, Chang Gung Memorial Hospital, Chiayi 61363, Taiwan, ROC.『email: ehuang@alumni.pitt.edu 
The non-framework surgery ${ }^{2}$ we modified from Friedman's ${ }^{9-11}$ reduced AHI and apneas but may increase the frequency or duration of hypopneas ${ }^{2-4}$. In patients with very severe OSA, although our earlier reports showed improvement of oxygen saturation after the surgery ${ }^{3,4}$, it is unclear whether this resulted from those without increased frequency or duration of hypopneas. There is a concern how the oxygen saturation would change in those having increased frequency or duration of hypopneas after the surgery. The aim of the study is to investigate whether hypopnea index or duration increase after the surgery might worsen oxygen saturation. Here we screened out patients with very severe OSA with increased frequency or duration of hypopneas after the surgery by multiplying hypopnea index (events/h) with mean hypopnea duration (s). Among them, we compared hypopnea index, mean hypopnea duration, obstructive-apnea index, mean obstructive-apnea duration, mean $\mathrm{SpO} 2$, minimal SpO2, and mean desaturation before and after the surgery. The results show that the reduction of frequency or duration of obstructive apneas may mask the negative effect of increased frequency or duration of hypopneas and improved the above oxygen saturation parameters.

\section{Materials and methods}

From 2015 to 2020, adult patients with very severe OSA that met these criteria were enrolled:

- Age 20 or older

- AHI equals to or over 60 events/h

- Refusal or unsuccessful of continuous positive airway pressure (CPAP)

- Received a one-stage multi-level sleep surgery with the modified Z-palatoplasty (ZPP) performed with onelayer closure and open partial tongue-base glossectomy ${ }^{2}$

- Underwent polysomnographies (PSGs) before and after the surgery with needed recordings, including mean $\mathrm{SpO} 2$, minimal $\mathrm{SpO} 2$, and mean desaturation

- Increased frequency or duration of hypopneas after the surgery.

We illustrated and detailed the surgery in the earlier works ${ }^{2,3}$. Each PSG was carried out overnight in the level 1 sleep laboratory of the authors' tertiary referral hospital by a certified technician and interpreted by a certified sleep physician. In a PSG, a hypopnea is defined as a decrease in airflow $\geq 30 \%$ for at least $10 \mathrm{~s}$ accompanied by either electroencephalography (EEG) signs of arousal or by a $4 \%$ or greater decrease in oxygen saturation ${ }^{12}$. An apnea is defined as the complete cessation of airflow for at least $10 \mathrm{~s}^{12}$. Increased frequency or duration of hypopneas is defined as having an increase in the product of hypopnea index (events/h) and mean hypopnea duration (s).

To further understand the impact of one sleep parameter, we conducted a paired t-test for each of AHI, hypopnea index, mean hypopnea duration, obstructive-apnea index, and mean obstructive-apnea duration. We scatter plotted and calculated the product of hypopnea index and mean hypopnea duration and that of obstructive-apnea index and mean duration of obstructive apnea, with a paired t-test to analyze the differences against no difference after the surgery. Then we scatter plotted and conducted a paired t-test for each of mean $\mathrm{SpO} 2$, minimal SpO2, and mean desaturation before and after the surgery. A Lilliefors test was performed to test the normality of differences before and after the surgery for all of the parameters mentioned above. A p value $<0.05$ indicated a statistical significance.

The statistical examinations were conducted in Matlab 9.4.0.813654 (MathWorks, Natick, Massachusetts, U.S.A.).

Ethical statements. The Institutional Review Board (IRB) of Chang Gung Medical Foundation, Taiwan, approved the study methods and protocols (IRB number: 202001198B0). We performed the study under Good Clinical Practice and the laws and regulations. As a retrospective cohort study, the IRB approved the waiver of the participants' consent.

\section{Results}

Fifteen men and two women with very severe OSA aged between 25 and 63 years were enrolled in this work. The average body mass index (BMI) was 28.9 with a standard deviation of $3.6 \mathrm{~kg} / \mathrm{m}^{2}$. A PSG was performed about 6 months ( $192 \pm 78$ days) after the surgery, when the anatomy was considered stable (as the period of 3 to 6 months was adopted in the literature, e.g., see $\left.{ }^{13,14}\right)$. The Lilliefors tests showed that the differences before and after the surgery for all of the tested parameters were normally distributed, except for the product difference of hypopnea index and mean hypopnea duration. Among these 17 patients, the surgery reduced AHI from $73.7 \pm 9.2$ (a standard deviation) to $45.3 \pm 15.1$ events $/ \mathrm{h}, \mathrm{p}<0.001$. The mean AHI reduction was 28.4 events $/ \mathrm{h}$, with a $95 \%$ confidence interval of 19.7 to 37.1 events $/ \mathrm{h}$. Although the mean BMI decreased $1.2 \mathrm{~kg} / \mathrm{m}^{2}(\mathrm{p}=0.044)$, with a $95 \%$ confidence interval of 0.04 to $2.42 \mathrm{~kg} / \mathrm{m}^{2}$, BMI change was not statistically correlated with AHI reduction $(\mathrm{r}=0.129, \mathrm{p}=0.621)$.

The preoperative hypopnea index ranged from 0.6 to 34.1 events/h. The postoperative hypopnea index ranged from 6.4 to 53 events/h. A paired t-test showed hypopnea index increased from a mean of $14.4 \pm 10.9$ events/h to $31.2 \pm 15.4$ events/h, $\mathrm{p}<0.001$. The mean increase of hypopnea index was 16.9 events/h, with a $95 \%$ confidence interval of 8.9 to 24.9 events/h. The preoperative mean hypopnea duration ranged from 16.3 to $31.5 \mathrm{~s}$. The postoperative mean hypopnea duration ranged from 21 to $68.7 \mathrm{~s}$. A paired t-test showed mean hypopnea duration increased from $24.0 \pm 6.1 \mathrm{~s}$ to $33.8 \pm 12.6 \mathrm{~s}, \mathrm{p}=0.011$. The mean increase of hypopnea duration was $9.7 \mathrm{~s}$, with a $95 \%$ confidence interval of 2.5 to $17.0 \mathrm{~s}$. 

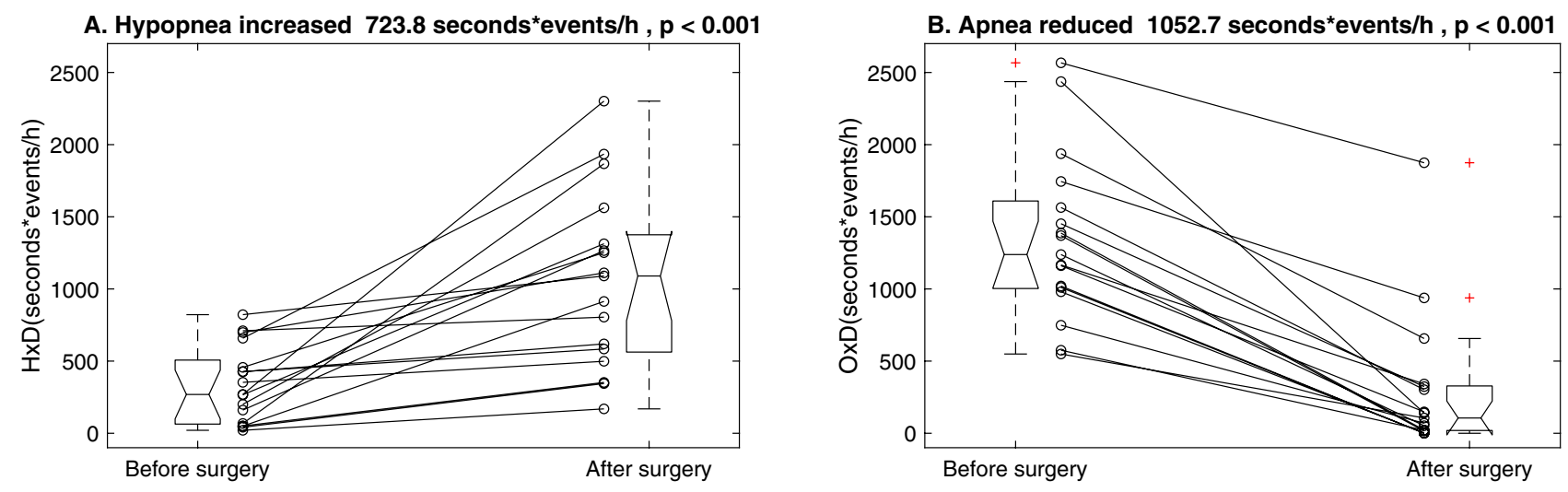

Figure 1. Individual product of (A) hypopnea index and mean hypopnea duration and (B) obstructive-apnea index and mean obstructive-apnea duration before and after the surgery. The surgery reduced the product on obstructive apnea but increased that on hypopnea.

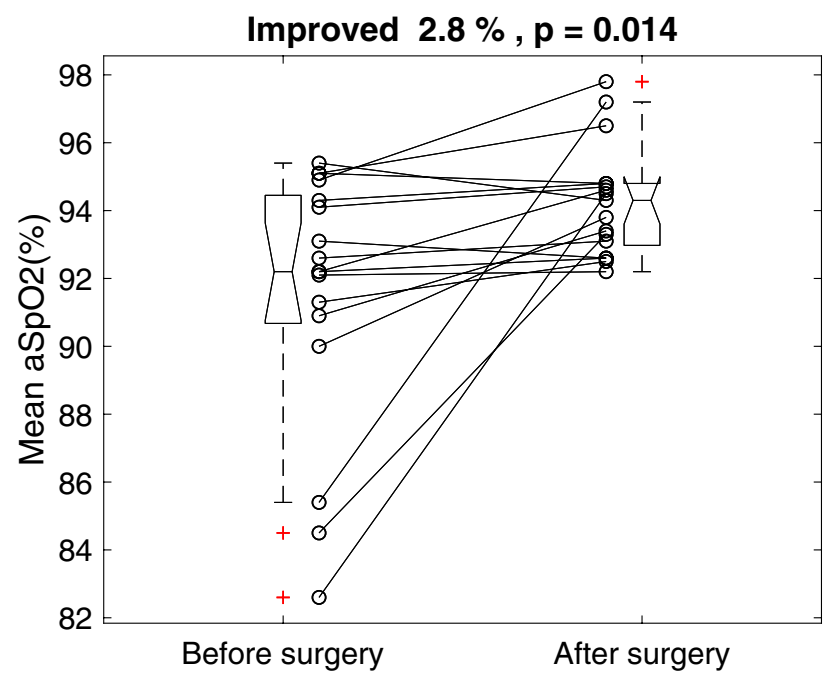

Figure 2. Individual mean oxyhemoglobin saturation of pulse oximetry ( $\mathrm{SpO} 2)$ before and after the surgery.

The preoperative obstructive-apnea index ranged from 16.2 to 73 events/h. The postoperative obstructiveapnea index ranged from 0 to 50.8 events/h. A paired t-test showed obstructive-apnea index reduced from a mean of $46.3 \pm 15.2$ events $/ \mathrm{h}$ to $10.2 \pm 14.0$ events $/ \mathrm{h}, \mathrm{p}<0.001$. The mean reduction of apnea index was 36.0 events $/ \mathrm{h}$, with a $95 \%$ confidence interval of 26.7 to 45.3 events/h. The preoperative mean obstructive-apnea duration ranged from 18.5 to $47.8 \mathrm{~s}$. The postoperative mean obstructive-apnea duration ranged from 0 to $40.2 \mathrm{~s}$. A paired $\mathrm{t}$-test showed mean obstructive-apnea duration decreased from $29.7 \pm 9.3 \mathrm{~s}$ to $21.1 \pm 11.6 \mathrm{~s}, \mathrm{p}=0.0015$. The mean reduction of apnea duration was $8.5 \mathrm{~s}$, with a $95 \%$ confidence interval of 3.8 to $13.3 \mathrm{~s}$.

Figure 1 shows the individual product of hypopnea index and mean hypopnea duration and that of obstructive-apnea index and mean obstructive-apnea duration before and after the surgery. The surgery increased the mean product on hypopnea from $334 \pm 265$ to $1058 \pm 612 \mathrm{~s}^{*}$ events/h. The average increase of the mean product on hypopnea was $724 \mathrm{~s}^{\star}$ events/h, with a $95 \%$ confidence interval of 404 to $1043 \mathrm{~s}^{\star}$ events/h. It reduced the mean product on obstructive apnea from $1348 \pm 574$ to $295 \pm 482 \mathrm{~s}^{\star}$ events/h. There is a probable floor effect on this reduction (panel B). The average reduction of the mean product on obstructive apnea was $1053 \mathrm{~s}^{\star}$ events/h, with a $95 \%$ confidence interval of 835 to $1270 \mathrm{~s}^{\star}$ events/h.

Figure 2 shows the individual mean $\mathrm{SpO} 2$ before and after the surgery. The surgery improved the average of the mean $\mathrm{SpO} 2$ by $2.8 \%$ (with a ceiling effect) from $91.5 \pm 3.9$ to $94.3 \pm 1.6 \%, \mathrm{p}=0.014$. The mean $\mathrm{SpO} 2$ improved $2.8 \%$, with a $95 \%$ confidence interval of 0.6 to $4.9 \%$

Figure 3 shows the individual minimal SpO2 before and after the surgery. The surgery improved mean minimal SpO2 by $7.5 \%$ from $68.6 \pm 12.2$ to $76.1 \pm 6.4 \%, \mathrm{p}=0.015$. The mean minimal $\mathrm{SpO} 2$ improved $7.5 \%$, with a $95 \%$ confidence interval of 1.7 to $13.4 \%$

Figure 4 shows the individual mean desaturation before and after the surgery. The surgery reduced mean desaturation by $5 \%$ from $11.6 \pm 5.5$ to $6.6 \pm 1.8 \%, \mathrm{p}=0.002$. The mean desaturation reduced $5.0 \%$, with a $95 \%$ confidence interval of 2.1 to $7.9 \%$ 


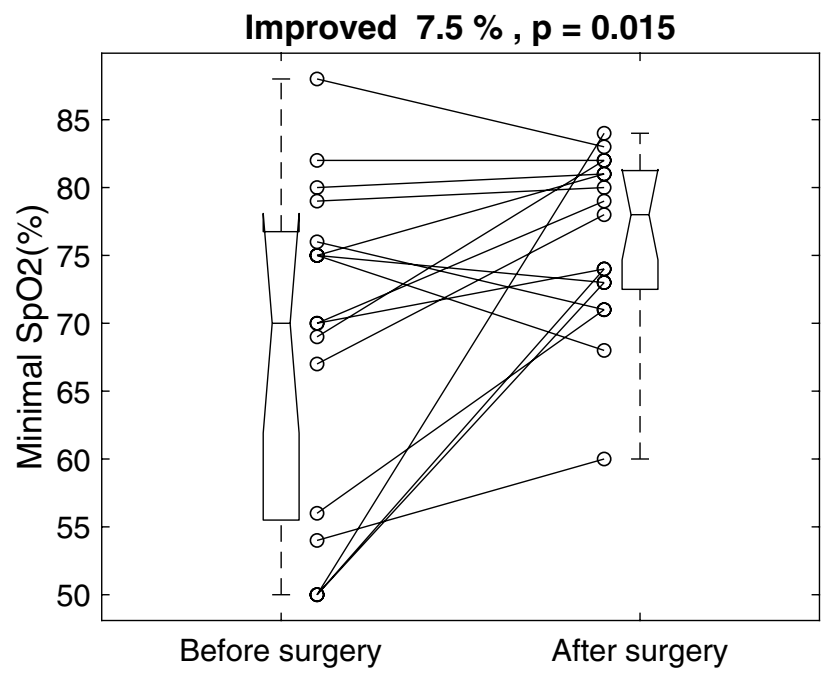

Figure 3. Individual minimal oxyhemoglobin saturation of pulse oximetry (SpO2) before and after the surgery.

\section{Reduced $5 \%, p=0.002$}

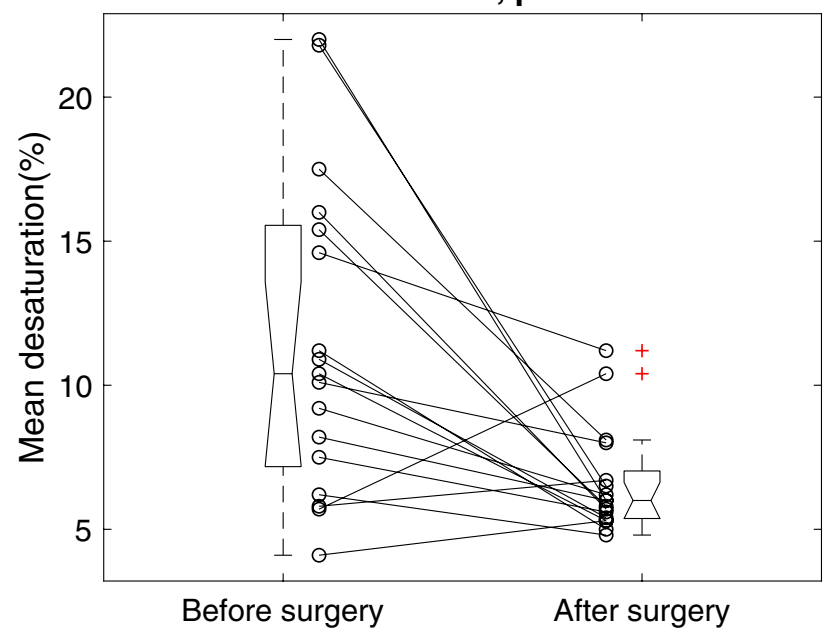

Figure 4. Individual mean desaturation before and after the surgery.

\section{Discussion}

In patients with very severe OSA with $\mathrm{AHI} \geq 60$ events/h and having increased frequency or duration of hypopneas after the non-framework surgery, the results show that the surgery improved oxygen saturation by reducing obstructive-apnea index (36.1 events/h) and duration (8.6 s/event), despite it increased hypopnea index (16.8 events/h) and duration ( $9.8 \mathrm{~s} /$ event). The surgery improved the average of the mean $\mathrm{SpO} 2$ by $2.8 \%$ (with a ceiling effect to a postoperative mean of $94.3 \%$ ), mean minimal SpO2 by $7.5 \%$, and mean desaturation by $5 \%$. There are very few detailed reports of hypopnea in patients with very severe OSA. The sample size of 17 in this study is small, although it is larger than 6 in Walker's ${ }^{15}, 9$ in Jacobowitz's ${ }^{16}, 10$ in Mickelson's ${ }^{17}$, and 11 in Vilaseca's ${ }^{18}$ for patients with very severe OSA. That limits the generalizability of the results. It needs future studies with a larger sample size to verify the observation. The results suggest that a sufficient shift from complete (apnea) to partial (hypopnea) cessation of airflow may mask the negative impact of the increase of hypopnea index or duration.

Respiratory disturbances can have unique characteristics even though the numbers are similar ${ }^{8}$. The AHI, which comprises the number of apneas and hypopneas, incorporates no information of the duration of these events. It is unlikely that an event lasting $10 \mathrm{~s}$ is physiologically equivalent to an event lasting 2 or $3 \min ^{19}$. Omission of event duration is another weakness of the AHI as a disease- or severity-defining metric for OSA ${ }^{19}$. One patient in the present study had a worse AHI from 62.6 to 77.6 after the surgery. But for the components, his obstructive-apnea index improved from 38.6 to 31.9 events/h, mean obstructive-apnea duration from 45.2 to $29.4 \mathrm{~s}$, mean $\mathrm{SpO} 2$ from 85.4 to $97.2 \%$, minimal SpO2 from 50 to $84 \%$, and mean desaturation from 21.8 to $6.5 \%$, despite the increase of AHI.

Deeper desaturations or longer breathing cessations may have more severe consequences than shorter and shallower ones ${ }^{20}$. Pulmonary artery hemodynamics (mean pulmonary artery pressure and right atrial pressure) 
are associated with duration of nocturnal desaturation (percentage of sleep time spent with oxygen saturation $<90 \%$ ) but not $\mathrm{AHI}^{21}$. If severity was measured by the product of duration and depth of the individual obstruction event, the severity of individual obstruction events (not the AHI number) is related to the increased mortality rate in severe obstructive sleep apnea ${ }^{22}$. These support that AHI may weigh less on disease severity than desaturation and that an adequate shift from apnea to hypopnea may reduce the severity of the disease.

\section{Conclusions}

The frequency or duration of hypopnea could increase in patients with very severe OSA with AHI $\geq 60$ events/h after a non-framework surgery. But the results showed that sufficient apnea reduction and the shift from apnea to hypopnea might mask the negative impact of the increase of hypopnea index or duration and improve postoperative mean $\mathrm{SpO} 2$, minimal SpO2, and mean desaturation.

Received: 30 September 2021; Accepted: 25 January 2022

Published online: 10 February 2022

\section{References}

1. Berry, R. B. et al. The AASM Manual for the Scoring of Sleep and Associated Events: Rules Terminology and Technical Specifications (American Academy of Sleep Medicine, 2020).

2. Huang, E. I., Kuo, C. L., Chou, Y. T., Lin, Y. C. \& Huang, S. Y. Modified Z-palatoplasty with one-layer closure in one-stage multilevel surgery for severe obstructive sleep apnea. Auris Nasus Larynx 45, 791-795. https://doi.org/10.1016/j.anl.2017.10.002 (2018).

3. Huang, E. I. et al. Increasing hypopnea in sleep breathing disturbance improves postoperative oxygen saturation in patients with very severe obstructive sleep apnea. Appl. Sci. 10, 6539 (2020).

4. Huang, E. I., Lin, Y.-C., Huang, S.-Y., Lin, C.-K. \& Lin, C.-M. Shifting and reducing breathing disturbance in patients with very severe obstructive sleep apnea by modified Z-palatoplasty with one-layer closure in one-stage multilevel surgery. Sci. Rep. https:// doi.org/10.1038/s41598-021-88074-1 (2021).

5. Sarac, S. \& Afsar, G. C. Effect of mean apnea-hypopnea duration in patients with obstructive sleep apnea on clinical and polysomnography parameter. Sleep Breath. Schlaf \& Atmung 24, 77-81. https://doi.org/10.1007/s11325-019-01870-y (2020).

6. Zhan, X., Fang, F., Wu, C., Pinto, J. M. \& Wei, Y. A retrospective study to compare the use of the mean apnea-hypopnea duration and the apnea-hypopnea index with blood oxygenation and sleep patterns in patients with obstructive sleep apnea diagnosed by polysomnography. Med. Sci. Monit. 24, 1887-1893. https://doi.org/10.12659/msm.909219 (2018).

7. Kulkas, A., Duce, B., Leppänen, T., Hukins, C. \& Töyräs, J. Severity of desaturation events differs between hypopnea and obstructive apnea events and is modulated by their duration in obstructive sleep apnea. Sleep Breath. 21, 829-835. https://doi.org/10.1007/ s11325-017-1513-6 (2017).

8. Yllmaz Durmaz, D. \& Güneş, A. Which is more important: The number or duration of respiratory events to determine the severity of obstructive sleep apnea?. Aging Male 23, 119-124. https://doi.org/10.1080/13685538.2019.1630062 (2019).

9. Friedman, M., Ibrahim, H. Z., Vidyasagar, R., Pomeranz, J. \& Joseph, N. J. Z-palatoplasty (ZPP): A technique for patients without tonsils. Otolaryngol. Head Neck Surg. 131, 89-100. https://doi.org/10.1016/j.otohns.2004.02.051 (2004).

10. Friedman, M. \& Schalch, P. Surgery of the palate and oropharynx. Otolaryngol. Clin. North Am. 40, 829-843. https://doi.org/10. 1016/j.otc.2007.04.010 (2007).

11. Friedman, M., Wilson, M. \& Kelley, K. Modification of Z-palatoplasty technique and review of five-year experience. Oper. Tech. Otolaryngol. Head Neck Surg. 23, 30-35. https://doi.org/10.1016/j.otot.2011.06.004 (2012).

12. Kapur, V. K. et al. Clinical practice guideline for diagnostic testing for adult obstructive sleep apnea: An American Academy of Sleep Medicine Clinical Practice Guideline. J. Clin. Sleep Med. 13, 479-504. https://doi.org/10.5664/jcsm.6506 (2017).

13. Li, H. Y. et al. Three-dimensional computed tomography and polysomnography findings after extended uvulopalatal flap surgery for obstructive sleep apnea. Am. J. Otolaryngol. 26, 7-11. https://doi.org/10.1016/j.amjoto.2004.06.006 (2005).

14. Hessel, N. S. \& de Vries, N. Results of uvulopalatopharyngoplasty after diagnostic workup with polysomnography and sleep endoscopy: A report of 136 snoring patients. Eur. Arch. Otorhinolaryngol. 260, 91-95. https://doi.org/10.1007/s00405-002-0511-9 (2003).

15. Walker, E. B., Frith, R. W., Harding, D. A. \& Cant, B. R. Uvulopalatopharyngoplasty in severe idiopathic obstructive sleep apnoea syndrome. Thorax 44, 205-208. https://doi.org/10.1136/thx.44.3.205 (1989).

16. Jacobowitz, O. Palatal and tongue base surgery for surgical treatment of obstructive sleep apnea: A prospective study. Otolaryngol. Head Neck Surg. 135, 264.e261. https://doi.org/10.1016/j.otohns.2006.03.029 (2006).

17. Mickelson, S. A. \& Rosenthal, L. Midline glossectomy and epiglottidectomy for obstructive sleep apnea syndrome. Laryngoscope 107, 614-619. https://doi.org/10.1097/00005537-199705000-00011 (1997).

18. Vilaseca, I., Morelló, A., Montserrat, J., Santamaría, J. \& Iranzo, A. Usefulness of uvulopalatopharyngoplasty with genioglossus and hyoid advancement in the treatment of obstructive sleep apnea. Arch. Otolaryngol.-Head Neck Surg. 128, 435-440. https:// doi.org/10.1001/archotol.128.4.435 (2002).

19. Punjabi, N. M. COUNTERPOINT: Is the apnea-hypopnea index the best way to quantify the severity of sleep-disordered breathing? No. Chest 149, 16-19. https://doi.org/10.1378/chest.14-2261 (2016).

20. Kulkas, A., Tiihonen, P., Julkunen, P., Mervaala, E. \& Töyräs, J. Novel parameters indicate significant differences in severity of obstructive sleep apnea with patients having similar apnea-hypopnea index. Med. Biol. Eng. Compu. 51, 697-708. https://doi.org/ 10.1007/s11517-013-1039-4 (2013).

21. Samhouri, B. et al. Pulmonary artery hemodynamics are associated with duration of nocturnal desaturation but not apneahypopnea index. J. Clin. Sleep Med. 16, 1231-1239. https://doi.org/10.5664/jcsm.8468 (2020).

22. Muraja-Murro, A. et al. The severity of individual obstruction events is related to increased mortality rate in severe obstructive sleep apnea. J. Sleep Res. 22, 663-669. https://doi.org/10.1111/jsr.12070 (2013).

\section{Author contributions}

E.I.H. conceptualized the study, analyzed the PSGs, ran analysis, and wrote the primary draft of the manuscript. S.-Y.H. and Y.-C.L. registered the patients, interpreted the data, and provided essential comments on the manuscript. C.-K.L., C.-M.L., Y.-C.H., C.-Y.H, and J.-A.S. registered the patients, read the PSGs, and revised the manuscript.

\section{Competing interests}

The authors declare no competing interests. 


\section{Additional information}

Correspondence and requests for materials should be addressed to E.I.H.

Reprints and permissions information is available at www.nature.com/reprints.

Publisher's note Springer Nature remains neutral with regard to jurisdictional claims in published maps and institutional affiliations.

(c) (1) Open Access This article is licensed under a Creative Commons Attribution 4.0 International License, which permits use, sharing, adaptation, distribution and reproduction in any medium or format, as long as you give appropriate credit to the original author(s) and the source, provide a link to the Creative Commons licence, and indicate if changes were made. The images or other third party material in this article are included in the article's Creative Commons licence, unless indicated otherwise in a credit line to the material. If material is not included in the article's Creative Commons licence and your intended use is not permitted by statutory regulation or exceeds the permitted use, you will need to obtain permission directly from the copyright holder. To view a copy of this licence, visit http://creativecommons.org/licenses/by/4.0/.

(C) The Author(s) 2022 